\title{
Algoritmos Rápidos para Estimar o Subespaço Sinal em Problemas de Posto Incompleto
}

M.I. CARDOSO ${ }^{1}$, F.S.V. BAZÁN ${ }^{2}$, Departamento de Matemática, Universidade Federal de Santa Catarina, Campus Universitário, 88010-970 Florianópolis, SC, Brasil.

Resumo. Apresentamos uma versão modificada do método de Lanczos para calcular o subespaço invariante associado aos maiores valores singulares de matrizes de Hankel de posto incompleto. O método utiliza recomeços implícitos para acelerar a convergência e efetua produtos matriz vezes vetor de maneira rápida. São apresentados resultados numéricos para ilustrar a potencialidade do método.

\section{Introdução}

A estimativa dos $d$-maiores valores singulares e correspondentes vetores singulares de uma matriz de Hankel $H$, com dimensão $M \times N$, e cujas entradas são amostras de sinais discretos, tem atraído muita atenção em diversas áreas, por exemplo, ressonância magnética nuclear, processamento de imagens, análise modal de estruturas mecânicas, etc. O subespaço gerado por esses vetores singulares é chamado subespaço sinal. Os sinais em estudo são descritos por:

$$
h(t)=r_{1} e^{s_{1} t}+r_{2} e^{s_{2} t}+\ldots+r_{d} e^{s_{d} t}, \quad t \geq 0,
$$

com $r_{i}, s_{i} \in \mathbf{C}, \operatorname{real}\left(s_{i}\right)<0, i=1, \ldots, d$. As matrizes de Hankel são do tipo:

$$
H=\left[\begin{array}{ccccc}
h_{j} & h_{j+1} & \cdots & h_{j+N-2} & h_{j+N-1} \\
h_{j+1} & h_{j+2} & \cdots & h_{j+N-1} & h_{j+N} \\
\vdots & \vdots & \cdots & \vdots & \vdots \\
h_{j+M-1} & h_{j+M} & \cdots & h_{j+M+N-3} & h_{j+M+N-2}
\end{array}\right]_{M \times N}
$$

onde $h_{j}=h(j \Delta t), j \geq 0$. Prova-se que $\operatorname{posto}(H)=d$, [1]. Na prática, as amostras disponíveis são da forma $\widetilde{h}_{j}=h_{j}+\epsilon_{j}$, onde os $\epsilon_{j}$ denotam o ruído nas amostras e assim, a matriz de Hankel $\widetilde{H}=H+E$ se comporta como sendo de posto completo.

$\mathrm{O}$ algoritmo mais conhecido para calcular valores e vetores singulares é o algoritmo proposto por Golub e Reinsch [6]. Porém, como este algoritmo calcula todos

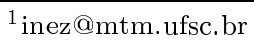

2 fermin@mtm.ufsc.br
} 
os valores singulares, ele pode ser muito demorado e, portanto, inconveniente em nosso caso, pois em geral $d \ll \min \{M, N\}$. Isso motiva o estudo de algoritmos iterativos para calcular decomposições espectrais parciais, as quais utilizam unicamente multiplicações matriz vezes vetor. Alguns algoritmos deste tipo são analisados em [5], onde os valores e vetores singulares de interesse são computados a partir do problema de autovalor associado a $\widetilde{H} * \widetilde{H}$, onde * denota a Hermitiana de $\widetilde{H}$.

Neste trabalho, apresentamos algoritmos para calcular o subespaço sinal, os quais utilizam Iterações de Lanczos e exploram multiplicações rápidas via FFT (Fast Fourier Transform) [5]. Introduzimos uma escolha inicial dos processos iterativos que garante convergência em no máximo $d+1$ iterações quando o sinal for livre de ruído, mostrando resultados numéricos que ilustram a validade desta propriedade, ainda no caso de dados com ruído, desde que a separação entre $\widetilde{\sigma}_{d}(\widetilde{H})$ e $\widetilde{\sigma}_{d+1}(\widetilde{H})$ seja bem notória. Apresentamos, também, resultados teóricos sobre valores singulares da matriz Hankel sem ruído, os quais explicam quando pode ser obtida a separação acima descrita em função do tamanho do ruído nos dados.

\section{Multiplicação Rápida}

Explorando a estrutura da matriz de Hankel e usando a Transformada Rápida de Fourier $(F F T)$, é possível diminuir o número de operações necessárias para efetuar multiplicações do tipo matriz-vetor. Sendo $H \in \mathbf{C}^{M \times N}$, através do uso da $F F T$, as conhecidas $M N$ multiplicações complexas necessárias para realizar essas multiplicações podem ser reduzidos para $O\left((M+N) \log _{2}(M+N)\right)$. Para mostrar como isto pode ser feito, vamos introduzir uma matriz circulante, denotada por $C$,

$$
C=\left[\begin{array}{lllll}
c_{0} & c_{N-1} & c_{N-2} & \ldots & c_{1} \\
c_{1} & c_{0} & c_{N-1} & \ldots & c_{2} \\
c_{2} & c_{1} & c_{0} & \ldots & c_{3} \\
\vdots & \vdots & \vdots & & \vdots \\
c_{N-1} & c_{N-2} & c_{N-3} & \ldots & c_{0}
\end{array}\right]_{N \times N}
$$

O seguinte teorema relaciona a transformada discreta de Fourier com matrizes circulantes, e é a base do processo de multiplicação rápida.

Teorema 1 Se $C$ é uma matriz circulante, então $C=F_{N}^{*} \Delta F_{N}$, onde $F_{N}$ é matriz de Fourier

$$
F_{N}=\frac{1}{\sqrt{N}}\left[\begin{array}{lllll}
1 & 1 & 1 & \ldots & 1 \\
1 & w^{1} & w^{2} & \ldots & w^{N-1} \\
1 & w^{2} & w^{4} & \ldots & w^{2(N-1)} \\
\vdots & \vdots & \vdots & \ldots & \vdots \\
1 & w^{N-1} & w^{2(N-1)} & \ldots & w^{(N-1)(N-1)}
\end{array}\right]
$$

$w=\exp (-2 \pi i / N)$ e $\Delta=\operatorname{diag}\left(F_{N} c\right)$, onde $c$ denota a primeira coluna da matriz $C$. 
Usando o teorema acima, obtemos uma forma de realizar o produto $y=C x$ usando duas transformadas de Fourier e uma transformada inversa de Fourier, as quais podem ser feitas eficientemente via o algoritmo da FFT. O uso da FFT é ainda melhor se a dimensão do vetor for uma potência de 2. Neste caso, o produto matriz vezes vetor utiliza $O\left(N \log _{2} N\right)$ multiplicações complexas. Observe ainda que, se for necessário calcularmos este produto para vários vetores, só precisamos calcular $F_{N} c$ uma vez, o que reduz o número de $F F T$ a serem calculadas.

Considere agora a matriz circulante $C$ definida por

$$
C=\left[\begin{array}{cccc}
h_{M} & h_{M+1} & h_{M+2} & \ldots \\
h_{M-1} & h_{M} & h_{M+1} & \ldots \\
\vdots & \vdots & \vdots & \ldots \\
h_{1} & h_{2} & h_{3} & \ldots \\
0 & h_{1} & h_{2} & \ldots \\
\vdots & \vdots & \vdots & \ldots \\
h_{M+N-1} & 0 & 0 & \cdots \\
h_{M+N-2} & h_{M+N-1} & 0 & \ldots \\
\vdots & \vdots & \vdots & \ldots \\
h_{M+1} & h_{M+2} & h_{M+3} & \cdots
\end{array}\right]
$$

e observe que se introduzimos $J=\left[e_{M} e_{M-1} \cdots e_{1}\right]$, então

$$
J C=\left[\begin{array}{ll}
\times & \times \\
H & \times
\end{array}\right]
$$

Para obter o produto $y=H x$, basta fazer $z=C \bar{x}$ usando a FFT de acordo com o teorema acima com $\bar{x}=\left[\begin{array}{ll}x & 0\end{array}\right]^{T}$ e, a seguir, tomar as $M$ primeiras componentes de

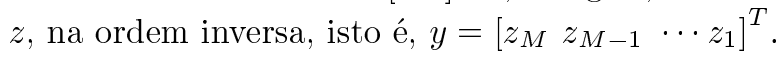

Algoritmo 1 Multiplicação Rápida.

1. Calcule $g=F_{N} c$

2. Calcule $z=F_{N} x$

3. Calcule $w=g . * z$

4. Calcule $y=F_{N}^{*} w$

onde $\left(.^{*}\right)$ denota a multiplicação ponto a ponto.

\section{Método de Lanczos com Recomeços Implícitos}

Existem várias técnicas para aproximar uma parte do espectro de uma matriz simétrica. Muitas delas, baseadas na projeção sobre subespaços de dimensão pequena, normalmente subespaços de Krylov, dentre as quais destaca-se o método de 
Lanczos. A seguir, apresentamos uma modificação do método de Lanczos, conhecida como o método de Lanczos com recomeços implícitos, onde os produtos matriz vezes vetor são feitos através do algoritmo da multiplicação rápida descrito anteriormente.

O método clássico de Lanczos está baseado no fato de que toda matriz Hermitiana, $A \in \mathbf{C}^{N \times N}$, pode ser transformada numa matriz tridiagonal $T$, num número finito de passos, através de transformações unitárias [6]:

$$
T=Q^{*} A Q
$$

Equacionando as $j$ primeiras colunas de (3.2) obtemos

$$
A Q_{j}=Q_{j} T_{j}+r_{j} e_{j}^{T}
$$

onde $r_{j}=\beta_{j} q_{j+1}, Q_{j}=\left(q_{1}, \ldots, q_{j}\right), Q_{j}^{*} Q_{j}=I$ e $T_{j}$ é o menor $j \times j$ de $T$

$$
T_{j}=\left[\begin{array}{ccccc}
\alpha_{1} & \beta_{1} & & & \\
\beta_{1} & \alpha_{2} & \beta_{2} & & \\
& \ddots & \ddots & \ddots & \\
& & \ddots & \ddots & \beta_{j-1} \\
& & & \beta_{j-1} & \alpha_{j}
\end{array}\right],
$$

com $\alpha_{i}=q_{i}^{*} A q_{i}$ e $\beta_{i}=\left\|r_{i}\right\|$. Os vetores $q_{i}$ são chamados vetores de Lanczos.

Similarmente, a equação (3.3) pode ser rescrita como

$$
r_{j}=\beta_{j} q_{j+1}=A q_{j}-\alpha_{j} q_{j}-\beta_{j-1} q_{j-1} .
$$

A partir de (3.4) é deduzida a recursão de Lanczos, a qual, dado um vetor inicial $q_{1}$, gera, a cada iteração, um novo vetor de Lanczos, e uma nova linha e coluna são adicionadas à matriz $T_{j}$. Se $\tau_{l}, g_{l}, \quad 1 \leq l \leq j$, são um par autovalor-autovetor de $T_{j}$, então os $\tau_{l}$, chamados valores de Ritz, aproximam os maiores autovalores de A, enquanto que os vetores $y_{l}=Q_{j} g_{l}$, chamados vetores de Ritz, aproximam os correspondentes autovetores [6].

Algoritmo 2 Método de Lanczos Básico.

Seja $A=\widetilde{H}^{*} \widetilde{H}$. Dados $r_{0} \neq 0, \beta_{0}=\left\|r_{0}\right\|$, para $j=1,2, \ldots$, faça:

1. $q_{j}:=r_{j-1} / \beta_{j-1}$

2. $u_{j}:=A q_{j}=H^{*}\left(H q_{j}\right)$

3. $r_{j}:=u_{j}-\beta_{j-1} q_{j-1}$

4. $\alpha_{j}:=q_{j}^{*} r_{j}$

5. $r_{j}:=r_{j}-\alpha_{j} q_{j}$

6. $\beta_{j}:=\left\|r_{j}\right\|$

7. Se desejar, calcule os valores de Ritz $\tau_{i}$ e vetores de Ritz $y_{i}$. Se a quantidade desejada de valores e vetores de Ritz convergiu, então pare. 
Uma deficiência do método é que, em aritmética finita, os vetores $q_{j}$ perdem a ortogonalidade. Com isso, são produzidas cópias de vetores de Ritz já calculados, tornando o processo lento. Uma maneira de resolver este problema, é fazer uma reortogonalização completa. Só que, neste caso, além do trabalho extra para a reortogonalização, é necessário manter todos os vetores de Lanczos armazenados. Esta idéia é chamada de Lanczos com reortogonalização completa. No outro extremo, tem-se o método de Lanczos sem reortogonalização, porém, com a exigência de uma análise mais detalhada das aproximações obtidas. Entre estes dois, estão os métodos que fazem uma reortogonalização parcial, ou reortogonalização seletiva, isto é , reortogonalizam somente quando há necessidade. Uma maneira de não deixar o número de vetores de Lanczos ficar muito grande, é recomeçar o processo após um número fixo de iterações, com um vetor inicial escolhido de maneira a aproveitar a estrutura da matriz $A$. Neste caso, a reortogonalização é possível, pois somente um número fixo de vetores de Lanczos é mantido na memória.

Em problemas onde não existe uma boa separação entre os autovalores desejados e os não desejados, normalmente são necessários muitos passos de Lanczos para convergir. Assim, a reortogonalização completa apresenta uma desvantagem, pois ela exige o armazenamento de todos os vetores de Lanczos. Uma forma de contornar este problema, é recomeçar as iterações após um número finito de passos, mantendo a quantidade de vetores a ser armazenada fixa e a reortogonalização é feita somente quando for necessário. Calvetti, Reichel e Sorensen desenvolveram um método de Lanczos com recomeços implícitos (IRL) [4], o qual é uma adaptação do método de Arnoldi com recomeços [8] para o caso Hermitiano. Este método força o vetor inicial a estar num subespaço invariante da matriz através de repetidas filtragens do vetor inicial e recomeçando as iterações implicitamente.

O teorema abaixo afirma que se $q_{1}$ for uma combinação linear de $d$ autovetores, então a recursão terminará em $d$ passos, com $r_{d}=0$. Além disto, a equação (3.4) força os vetores de Lanczos a estarem no subespaço gerado por estes $d$ autovetores. Assim, uma boa escolha de vetor inicial, é como tal combinação.

Teorema 2 Seja $A_{n \times n}$ uma matriz simétrica e seja $A Q_{d}-Q_{d} T_{d}=r_{d} e_{d}^{T}$, com $T$ simétrica não reduzida, passo d da fatoração de Lanczos de A. Então, $r_{d}=0$ se, e somente se, $q_{1}=V_{d} x$, onde

$$
A V_{d}=V_{d} \Lambda_{d}
$$

com $V_{d}^{*} V_{d}=I_{d}$ e $\Lambda_{d}$ uma matriz diagonal de ordem $d$.

Demonstração. $(\Rightarrow)$ Suponha que $r_{d}=0$. Então, $A Q_{d}=Q_{d} T_{d}$. Seja $T_{d} G_{d}=G_{d} \Lambda_{d}$ a decomposição autovalor-autovetor de $T_{d}, G_{d}^{*} G_{d}=I_{d}$. Chamando $V_{d}=Q_{d} G_{d}$, então $A Q_{d} G_{d}=Q_{d} G_{d} \Lambda_{d}$, isto é, $A V_{d}=V_{d} \Lambda_{d}$. Além disso, posto $\left(V_{d}\right)=d$ e $q_{1}=Q_{d} e_{1}=Q_{d} G_{d} G_{d}^{*} e_{1}=V_{d} x, \operatorname{com} x=G_{d}^{*} e_{1}$.

$(\Leftarrow)$ Suponha, agora, que $A V_{d}=V_{d} \Lambda_{d}, \operatorname{rank}\left(V_{d}\right)=d$ e $q_{1}=V_{d} x$. Então, para qualquer inteiro $m>0, A^{m} V_{d}=V_{d} \Lambda_{d}^{m}$ e, assim, para todo $m$,

$$
A^{m} q_{1}=A^{m} V_{d} x=V_{d} \Lambda_{d}^{m} x \in \mathcal{R}\left(V_{d}\right)
$$


Consequentemente, $\operatorname{dim}\left(\mathcal{K}^{d+1}\left(A, q_{1}\right)\right) \leq \operatorname{posto}\left(V_{d}\right)=d$. Como $T_{d}$ é não reduzida, usando uma pequena modificação do Teorema 7.4.3 de Golub e Van Loan [6], temos que $\operatorname{dim}\left(\mathcal{K}^{d+1}\left(A, q_{1}\right)\right)=d$ e, então, $r_{d}=0$.

Fixando o número de passos Lanczos em $d+p$, onde $p$ em geral não é muito maior do que $d$, sendo menor em alguns casos, obtem-se:

$$
A Q_{d+p}=Q_{d+p} T_{d+p}+\beta_{d+p} Q_{d+p} e_{d+p}^{T} .
$$

O $I R L$ aplica o algoritmo $Q R$ com shifts a esta decomposição truncada de $A$ da seguinte maneira: Seja $\mu_{1}$ um shift, calcule a decomposição $Q R$ de $T_{d+p}-\mu_{1} I=$ $X_{1} R_{1}$, onde $X_{1}, R_{1} \in \mathbf{C}^{(d+p) \times(d+p)}, X_{1}^{*} X_{1}=I_{d+p}$ e $R_{1}$ é triangular superior. Partindo agora de $T_{d+p}^{+}=X_{1}^{*} T_{d+p} X_{1}$, aplique novamente o algoritmo $Q R$ com shift $\mu_{2}$. Desta forma, após $p$ shifts obtém-se $A Q_{d+p}^{+}=Q_{d+p}^{+} T_{d+p}^{+}+\beta_{d+p} q_{d+p+1} e_{d+p}^{T} \hat{X}$, onde $Q_{d+p}^{+}=Q_{d+p} \hat{X}, T_{d+p}^{+}=\hat{X}^{*} T_{d+p} \hat{X}$ e $\hat{X}=X_{1} X_{2} \cdots X_{p}$. Analisando as $d$ primeiras colunas desta decompoisição obtém-se: $A Q_{d}^{+}=Q_{d}^{+} T_{d}^{+}+\beta_{d}^{+} q_{d+1}^{+} e_{d}^{T}$. Partindo agora desta nova decomposição de Lanczos e calculando $p$ passos de Lanczos, voltamos a mesma situação da equação (3.6). Portanto, o IRL consiste em aplicar alternadamente $p$ passos Lanczos e $p$ shifts.

Os $p$ passos $Q R$ aplicados desempenham um papel de filtragem do vetor inicial, fazendo com que este esteja num subespaço invariante apropriado, $\operatorname{span}\left(V_{d}\right)$. O lema abaixo, extraído de [4], sugere que para eliminar o conjunto de autovalores não desejados de $T_{d+p}$, deve-se escolher os $p$ shifts $\mu_{j}$ como sendo os autovalores que estão na parte não desejada do espectro. Segundo Calvetti [4], estes shifts são chamados de shifts exatos.

Lema 1 Seja $\lambda\left(T_{d+p}\right)=\left\{\tau_{1}, \ldots, \tau_{d}\right\} \bigcup\left\{\mu_{1}, \ldots, \mu_{p}\right\}$ uma partição disjunta do espectro de $T_{d+p}$ e seja

$$
T_{d+p}^{+}=\hat{X}^{*} T_{d+p} \hat{X},
$$

onde $\hat{X}=X_{1} X_{2} \ldots X_{p}$ e $X_{j}$ é implicitamente determinado pelo shift $\mu_{j}$. Seja $\beta_{j}$ o elemento da posição $(j+1)$ da subdiagonal de $T_{d+p}^{+}$. Se $\beta_{j} \neq 0$ para $1 \leq j<d$, então $\beta_{d}=0 e$

$$
T_{d+p}^{+}=\left[\begin{array}{ll}
T_{d}^{+} & 0 \\
0 & \hat{T}_{p}
\end{array}\right]
$$

onde $\lambda\left(T_{d}^{+}\right)=\left\{\tau_{1}, \ldots, \tau_{d}\right\}$ e $\lambda\left(\hat{T}_{p}\right)=\left\{\mu_{1}, \ldots, \mu_{p}\right\}$. Além disso,

$$
q_{1}^{+}=Q_{d+p} \hat{X} e_{1}=\sum_{j=1}^{d} \eta_{j} y_{j},
$$

onde $\eta_{j} \in C$ e $y_{j}$ é o vetor de Ritz associado ao valor de Ritz $\tau_{j}$, isto é, $y_{i}=Q_{d+p} g_{j}$ com $T_{d+p} g_{j}=g_{j} \tau j$ para $1 \leq j \leq d$.

A demonstração do Lema 1 pode ser encontrada em [5]. O lema mostra que a escolha dos shifts exatos, é matematicamente equivalente a recomeçar as iterações com 
um vetor inicial, que é uma combinação linear dos vetores de Ritz de $A$ correspondentes aos $d$ autovalores desejados, isto é, como uma aproximção de uma combinação linear dos $d$ autovetores. O lema, porém, não apresenta nenhuma informação sobre a razão de convergência. Em [4], encontra-se uma discussão sobre outro tipo de shifts. Antes de discutir a convergência do método apresentamos o algoritmo IRL para aproximar os $d$ maiores autovalores. Assume-se que $\tau_{1} \geq \tau_{2} \geq \ldots \geq \tau_{d+p}$.

Algoritmo 3 Método de Lanczos Recomeços Implícitos e Shifts Exatos (IRL-ES). Seja $A=\widetilde{H}^{*} \widetilde{H}$. Dados $r_{0} \neq 0, \beta_{0}=\left\|r_{0}\right\|$ :

1. Faça $d+p$ passos usando o Algoritmo 2 (reortogonalizando quando necessário)

Repita

2. Calcule a decomposição autovalor-autovetor de $T_{d+p}$ e teste a convergência de $\beta_{(d+p) i}$

3. Se a quantidade desejada de pares de Ritz convergiu, calcule os vetores de Ritz e pare.

4. Aplique o algoritmo $Q R$ com shifts $\mu=\tau_{j}, j=d+1, \ldots, d+p$

5 Faça p passos de Lanczos, começando de (3.7), e volte para o passo 2 acima.

Nossa discussão sobre a convergência dos valores de Ritz está baseada no trabalho de Sorensen [10], onde é mostrado que à medida em que ocorrem as iterações, os autovalores de $T_{d}$ convergem para certos limites. Partindo disto, mostramos que estes limites são os autovalores de $A$ e, ainda mais, que são os $d$ maiores. Seja $l$ o número da iteração. Assim, $q_{1}^{(l)}$ representa o vetor inicial na iteração $l$ e após $p$ passos de Lanczos, tem-se

$$
A Q_{d+p}^{(l)}-Q_{d+p}^{(l)} T_{d+p}^{(l)}=r_{d+p}^{(l)} e_{d+p}^{T}
$$
Seja $T_{d+p}^{(l)}=\left[\begin{array}{ll}T_{d}^{(l)} & \beta_{d}^{(l)} e_{d} e_{1}^{T} \\ \beta_{d}^{(l)} e_{1} e_{d}^{T} & \widetilde{T}^{(l)}\end{array}\right]$ com autovalores $\tau_{1, l+1}>\ldots>\tau_{d, l+1}>$
$\mu_{1, l+1}>\ldots>\mu_{p, l+1}$.

Aplicando $p$ shifts, temos

$$
\hat{X}^{(l) H} T^{(l)} \hat{X}^{(l)}=\left[\begin{array}{ll}
T_{d}^{(l+1)} & 0 \\
0 & \hat{T}_{p}
\end{array}\right], Q_{d}^{(l+1)}=\left(Q_{d+p}^{(l)} \hat{X}^{(l)}\right)\left[\begin{array}{l}
I_{d} \\
0
\end{array}\right],
$$

onde $\hat{X}^{(l)}=X_{1}^{(l)} \ldots X_{p}^{(l)}$ são contruídos aplicando os shifts $\mu_{1, l+1}, \ldots, \mu_{p, l+1}$.

Para mostrar que o limite para o qual as sequências $\left\{\tau_{j l}\right\}_{l=1}^{\infty}$ convergem são autovalores de $A$, vamos precisar de um lema auxiliar, o qual é enunciado a seguir: Lema 2 Seja $M=\left[\begin{array}{ll}T & \beta e_{d} \\ \beta e_{d}^{T} & \alpha\end{array}\right]$ uma matriz simétrica tridiagonal. Então, as raízes da equação

$$
\beta^{2} e_{d}^{T}(T-\lambda I)^{-1} e_{d}=\alpha-\lambda
$$

são autovalores de $M$. 
Demonstração. A demonstração segue do cálculo do polinômio característico de M, ou veja $[9]$.

Seja $T=G \Lambda G^{*}$ a decomposição autovalor-autovetor de $T$. Como $(T-\lambda I)^{-1}=$ $G(\Lambda-\lambda I)^{-1} G^{*}$, substituindo na equação (3.8) tem-se:

$$
\beta^{2} \sum_{j=1}^{d} \frac{\eta_{j}^{2}}{\left(\tau_{j}-\lambda\right)}=\alpha-\lambda
$$

onde $\left(\eta_{1}, \eta_{2}, \cdots, \eta_{d}\right)=e_{d}^{T} G$ e $\Lambda=\operatorname{diag}\left(\tau_{1}, \cdots, \tau_{d}\right)$. Da equação (3.9), pode-se concluir que nenhum autovalor de $M$ é igual a $\tau_{i}, 1 \leq i \leq d$. Usando a propriedade do interlacing, veja Glolub [6, pag. 396], tem-se:

$$
\hat{\mu}<\tau_{d}<\widetilde{\tau}_{d}<\tau_{d-1}<\ldots \tau_{1}<\widetilde{\tau}_{1}
$$

onde $\left\{\widetilde{\tau}_{1}, \ldots, \widetilde{\tau}_{d}, \hat{\mu}\right\}$ são autovalores de $M$. Se os elementos da subdiagonal de $T$ são todos não nulos, então os $\tau_{j}$ são distintos (veja [9], capítulo 7) e nenhum dos $\eta_{j}$ é zero.

Lema 3 Seja $T_{d}^{(l)}=G^{(l)} \Lambda^{(l)} G^{(l) H} e\left(\eta_{1}^{l}, \eta_{2}^{l}, \ldots, \eta_{d}^{l}\right)=e_{d}^{T} G^{(l)}$. Assuma que $\tau_{j}$ são disjuntos, onde $\tau_{j l} \rightarrow \tau_{j}$. Então,

$$
\beta_{d}^{l} \eta_{j}^{l} \rightarrow 0, \text { quando } l \rightarrow \infty, j=1, \ldots, d
$$

e, como uma consequência,

$$
\left\|A Q^{(l)} g_{j}^{(l)}-Q^{(l)} g_{j}^{(l)} \tau_{j l}\right\|=\left|\beta^{(l)} \eta_{j}^{l}\right| \rightarrow 0
$$

onde $g_{j}^{l}=G^{(l)} e_{j}$ para $j=1, \ldots d$.

Demonstração. Considere a submatriz $(d+1) \times(d+1)$ principal de $T_{d+p}^{(l)}$

$$
M=\left[\begin{array}{ll}
T_{d}^{(l)} & \beta_{d}^{(l)} e_{d} \\
\beta_{d}^{(l)} e_{d}^{T} & \alpha^{(l)}
\end{array}\right]
$$

Do Lema 2, segue que os $d$ maiores autovalores $\hat{\tau}_{j l}$ de $M^{(l)}$ satisfazem

$$
\tau_{j l}<\hat{\tau}_{j l}<\tau_{j, l+1}
$$

onde a última desigualdade surge de $p-1$ aplicações adicionais do Lema 2. Além disso, através de manipulações algébricas da equação (3.9), obtém-se:

$$
\left(\beta \eta_{j}\right)^{2}=\left(\tau_{j}-\lambda\right)\left[\frac{(\alpha-\lambda)-\beta^{2} \sum_{i=j+1}^{d} \frac{\eta_{j}^{2}}{\left(\tau_{i}-\lambda\right)}}{1+\sum_{i=1}^{j-1} \frac{\eta_{i}^{2}\left(\tau_{j}-\lambda\right)}{\eta_{j}^{2}\left(\tau_{i}-\lambda\right)}}\right],
$$


para qualquer raíz $\lambda$. Substituindo da matriz $T_{d}^{(l)}$ as quantidades apropriadas indexadas por $l$ e fazendo $\lambda=\hat{\tau}_{j l}$, tem-se:

$$
\left(\beta_{d}^{(l)} \eta_{j}^{(l)}\right)^{2}<\left|\left(\tau_{j l}-\hat{\tau}_{j l}\right)\right|\left|\left(\alpha^{(l)}-\hat{\tau}_{j l}\right)-\beta_{d}^{(l)^{2}} \sum_{i=j+1}^{d} \frac{\eta_{j}^{(l)^{2}}}{\left(\tau_{i l}-\hat{\tau}_{j l}\right)}\right| .
$$

Observe que, como $\hat{\tau}_{j l}>\tau_{j l}>\tau_{i l}$ para $i=1,2, \ldots, j-1$, então o denominador na equação (3.11) é maior do que 1.

A hipótese de que o os limites $\tau_{j}$ são distintos implica que

$$
\left|\left(\alpha^{(l)}-\hat{\tau}_{j l}\right)-\beta_{d}^{(l)^{2}} \sum_{i=j+1}^{d} \frac{\eta_{j}^{(l)^{2}}}{\left(\tau_{i l}-\hat{\tau}_{j l}\right)}\right|,
$$

tem limites finitos para cada $j$. Assim, para $l$ suficientemente grande, existe uma constante positiva $K$ tal que

$$
\left(\beta_{d}^{(l)} \eta_{j}^{(l)}\right)^{2}<K\left|\left(\tau_{j l}-\hat{\tau}_{j l}\right)\right|<K\left|\left(\tau_{j l}-\tau_{j, l+1}\right)\right| \rightarrow 0, \text { quando } l \rightarrow \infty,
$$

pois cada sequência $\left\{\tau_{j l}, l=1,2, \cdots\right\}$ é uma sequência crescente convergente, para cada $j=1,2, \cdots, d$.

Como os $\tau_{j l}$ convergem para os autovalores de $A$, falta mostrar que estes são os $d$ maiores. Observe que o vetor inicial $q_{1}^{(l)}$ pode ser escrito como

$$
q_{1}^{(l)}=\frac{\Psi_{l}(A) q_{1}}{\left\|\Psi_{l}(A) q_{1}\right\|}
$$

onde $\psi_{i}(\lambda)=\nu_{i} \prod_{k=1}^{p}\left(\lambda-\mu_{k, i}\right), \Psi_{l}(\lambda)=\prod_{i=1}^{l} \psi_{i}(\lambda)$ e $q_{1}$ é o vetor inicial original.

Teorema 3 Suponha que o vetor inicial $q_{1}$ satisfaz $v_{j}^{*} q_{1}=\gamma_{j} \neq 0$ para $j=1, \ldots, d$, onde $v_{j}$ é o autovetor de $A$ associado ao autovalor $\lambda_{j}$ com os autovalores de $A$ listados em ordem decrescente. Seja $\beta_{i}^{(l)}$ o elemento da posição $i$ da subdiagonal de $T_{d}^{(l)}$ e assuma que $\beta_{i}^{(l)}>\epsilon>0$ para todo $i, l$. Então, as sequências

$$
\tau_{j l} \rightarrow \tau_{j}=\lambda_{j} \text { quando } l \rightarrow \infty .
$$

Demonstração. A hipótese de $\beta_{i}^{(l)}>\epsilon>0$ assegura que a separação dos $\tau_{j l}$ é uniforme para todo $l$, de forma que os limites $\tau_{j}$ são distintos. Isto implica que cada $\tau_{j}$ é um autovalor de $A$. Além disso, essa hipótese implica em uma limitação inferior uniforme de $\left|\eta_{j}^{(l)}\right|$ e então, $\beta_{d}^{(l)} \rightarrow 0$. Estas observações seguem do Lema 2.

Seja $\hat{p}_{d}^{(l)}(\lambda)=\prod_{i=1}^{d}\left(\lambda-\tau_{i l}\right)$ o polinômio característico de $T_{d}^{(l)}$ e seja $\hat{p}_{d}(\lambda)=$ $\prod_{i=1}^{d}\left(\lambda-\tau_{i}\right)$ o polinômio limite de $\hat{p}_{d}^{(l)}$. Então,

$$
\left\|\hat{p}_{d}^{(l)}(A) q_{1}^{(l)}\right\|=\prod_{j=1}^{d} \beta_{d}^{(l)} \rightarrow 0
$$


e, portanto

$$
\hat{p}_{d}^{(l)}\left(\lambda_{j}\right) \gamma_{j}^{(l)}=v_{j}^{*} \hat{p}_{d}^{(l)}(A) q_{1}^{(l)} \rightarrow 0
$$

Assim,

$$
\hat{p}_{d}\left(\lambda_{j}\right)=0 \text { ou } \gamma_{j}^{(l)} \rightarrow 0
$$

para todo $j=1, \ldots, n$. Isto significa que $n-d$ dos coeficientes da expansão de $\gamma_{j}^{(l)}$ tendem para 0 quando $l \rightarrow \infty$. Além disso, como cada seqüência $\left\{\tau_{j}, l=\right.$ $1,2, \ldots\}$ é convergente para $j=1, \cdots, d$, temos que os $d$ coeficientes da expansão, correspondentes aos autovalores $\tau_{j}$, devem ser todos limitados inferiormente por 0 pela hipótese $\beta_{i}^{(l)}>\epsilon>0$ para todo $j=1,2, \ldots, d-1$ e $l$.

Suponha agora que $\lambda_{j 1}=\tau_{1}<\lambda_{1}$. Então, o coeficiente da expansão é

$$
\gamma_{j 1}^{(l)}=v_{j 1}^{*} q_{1}^{(l)}=v_{j 1}^{*} \frac{\Psi_{l}(A) q_{1}}{\left\|\Psi_{l}(A) q_{1}\right\|}=\frac{\Psi_{l}\left(\tau_{1}\right) v_{j 1}^{*} q_{1}}{\left\|\sum_{i=1}^{n} \gamma_{i} \Psi_{l}\left(\lambda_{i}\right) v_{i}\right\|}=\frac{\gamma_{j 1} \Psi_{l}\left(\tau_{1}\right)}{\sqrt{\sum_{i=1}^{n} \gamma_{i}^{2} \Psi_{l}^{2}\left(\lambda_{i}\right)}},
$$

onde $v_{j 1}$ representa o autovetor de $A$ associado ao autovalor $\tau_{1}$ e $q_{1}=\sum_{i=1}^{n} \gamma_{i} v_{i}$, $\operatorname{com} v_{i}^{*} v_{j}=0$ quando $i \neq j$.

Assim,

$$
\left(\gamma_{j 1}^{(l)}\right)^{2}=\frac{\left(\gamma_{j 1} \Psi_{l}\left(\tau_{1}\right) / \Psi_{l}\left(\lambda_{1}\right)\right)^{2}}{\gamma_{1}^{2}+\sum_{i=2}^{n} \gamma_{i}^{2} \Psi_{l}^{2}\left(\lambda_{i}\right) / \Psi_{l}^{2}\left(\lambda_{1}\right)} \leq\left(\frac{\gamma_{j 1} \Psi_{l}\left(\tau_{1}\right)}{\gamma_{1} \Psi_{l}\left(\lambda_{1}\right)}\right)^{2},
$$

onde os $\gamma_{j}$ são os coeficientes da expansão de $q_{1}$. Agora, as raízes $\mu_{i l}$ dos polinômios filtros satisfazem $\lambda_{n} \leq \mu_{i l}<\tau_{1 l} \leq \tau_{1}<\lambda_{1}$, de forma que

$$
\frac{\Psi_{l}\left(\tau_{1}\right)}{\Psi_{l}\left(\lambda_{1}\right)}=\prod_{k=1}^{l}\left(\prod_{i=1}^{p}\left(\frac{\tau_{1}-\mu_{i k}}{\lambda_{1}-\mu_{i k}}\right)\right) \leq\left(\frac{\tau_{1}-\lambda_{n}}{\lambda_{1}-\lambda_{n}}\right)^{l p} \rightarrow 0
$$

pois $\left(\tau_{1}-\lambda_{n}\right) /\left(\lambda_{1}-\lambda_{n}\right)<1$. O que é uma contradição. Concluímos, então, que $\tau_{1}=\lambda_{1}$. Um argumento similar pode ser feito para cada $j$ nos casos $\tau_{j}<\lambda_{j}$ e isso conclui a demonstração.

Até este ponto, analisamos a convergência do IRL, porém não foi dito nada a respeito da taxa de convergência. A seguir, apresentamos alguns resultados que descrevem condições sob os quais obtém-se convergência rápida.

Lema 4 Seja $A=B+\tau I$ uma matriz Hermitiana $M \times M$, onde $B$ é Hermitiana semi-positiva definida, $\operatorname{posto}(B)=d e \tau>0$. Se, os $d$ autovetores associados aos $d$ maiores autovalores são distintos, e se $q_{0}$ não é ortogonal a nenhum destes $d$ autovetores e ortogonal a no mínimo um autovetor associado a um autovalor não desejado, então $\operatorname{dim}\left(\mathcal{K}^{d+1}\left(A, q_{0}\right)\right)=d+1$, ou seja, o método de Lanczos converge em no máximo $d+1$ passos.

Demonstração. A prova deste lema decorre da propriedade: $\mathcal{K}^{m}\left(A, q_{o}\right)=$ $\mathcal{K}^{m}\left(A-\tau I, q_{o}\right)=\mathcal{K}^{m}\left(B, q_{o}\right)$ e $\operatorname{dim}\left(\mathcal{K}^{m}\left(B, q_{o}\right)=d+1\right.$, para todo $m>d[12]$. 
Outro resultado importante em relação a convergência do método de Lanczos aplicado ao cálculo dos $d$ maiores valores singulares, é que a taxa de convergência depende da separação entre $\widetilde{\sigma}_{d}$ e $\widetilde{\sigma}_{d+1}$ (ver Teorema 6.4.1 em [7]): quanto maior a separação, maior é a taxa de convergência. Considerando que, em nosso caso, $\widetilde{H}=H+E$ e posto $(H)=d$, e usando teoria de perturbação de valores singulares, segue que $\widetilde{\sigma}_{d+1} \leq\|E\|$ e que uma melhor separação entre $\widetilde{\sigma}_{d}$ e $\widetilde{\sigma}_{d+1}$ ocorre quando $\|E\|<\sigma_{d}[6]$. Isto mostra que o tamanho do $\sigma_{d}$, como uma função da dimensão do problema e das características do sinal, desempenha um papel importante na convergência do método. O seguinte teorema fornece uma estimativa para $\sigma_{d}$ no caso em que são utilizadas matrizes quadradas.

Teorema 4 Seja $H$ a matriz de Hankel $M \times M$, descrita em (1.1) com $j=0$. Defina $z_{l}=e^{s_{l} \Delta t}$ e $\delta=\min _{l \neq k}\left|z_{l}-z_{k}\right|, 1 \leq l, k \leq d$. Então, um limitante inferior para $\sigma_{d}$ satisfaz

$$
\sigma_{d} \geq K\left[1+\frac{D_{M}^{2}}{(d-1) \delta^{2}}\right]^{1-d}\left(1-e^{-2 \alpha \Delta t M}\right)
$$

onde

$$
K=\frac{1}{\rho} \frac{1}{\left(1-e^{-2 \alpha \Delta t}\right)}, \quad \alpha=\min \left|\operatorname{real}\left(s_{l}\right)\right|, \quad \rho=\min \left|r_{l}\right|,
$$

com $D_{M}^{2}$ uma constante que depende do tamanho dos $z_{l}$ e $M$.

Demonstração. Primeiro observamos que a matriz de Hankel pode ser rescrita como

$$
H=W R W^{T},
$$

onde $W$ é uma matriz de Vandermonde $M \times d$, com entradas $W(i, j)=z_{j}^{i-1}$ e $R=\operatorname{diag}\left(r_{1}, \ldots, r_{d}\right)$. A seguir, usando propriedades de valores singulares, segue que

$$
\sigma_{d} \geq \sigma_{d}(R) \sigma_{d}(W)^{2}
$$

A demonstração termina aplicando o Teorema 3.2 de Bazan [3]

Em [3], pode ser encontrada uma expressão para $D_{M}$, onde é mostrado que $D_{M}^{2} \approx 0$ quando $M$ é suficientemente grande e $\left|z_{l}\right| \approx 1$, o que é muito freqüente em aplicações envolvendo processamento de sinais. Em [2], é analisado o comportamento do limitante dado em 3.13 para matrizes de Hankel retangulares. O resultado dessa análise é que a estimativa será melhor quando a matriz de Hankel for quadrada.

O teorema sugere ainda que $\sigma_{d}$ deve crescer com a dimensão de $H$ e que a estimativa melhora quando os $z_{l}$ ficam perto do círculo unitário no plano complexo, porém, não muito próximos uns dos outros. Portanto, devemos utilizar matrizes de Hankel quadradas com a maior dimensão possível a fim de que $\sigma_{d}$ possa vencer o tamanho da pertubação $\|E\|$, possibilitando, assim, uma boa separação entre $\widetilde{\sigma}_{d}$ e $\widetilde{\sigma}_{d+1}$.

Dos resultados obtidos do Lema 4 e Teorema 4, deduzimos que para obter convergência rápida do método de Lanczos no cálculo dos $d$ maiores valores singulares 
da matriz de Hankel $\widetilde{H}$ e correspondente subespaço invariante, deve-se ter $\|E\|<\sigma_{d}$, a dimensão da matriz de Hankel deve ser a maior possível e o vetor inicial $q_{0}$ deve ter fortes componentes no subespaço de interesse. Quando isto é satisfeito, pode-se esperar que a convergência seja rápida. Na seção seguinte apresentaremos um exemplo numérico que mostra que se $\|E\| \ll \sigma_{d}$, então o método de Lanczos converge em pouco mais de $d$ iterações, desde que o vetor inicial seja escolhido adequadamente.

Finalizando esta seção, apresentamos uma escolha de vetor inicial para o método de Lanczos, a qual mostra resultados melhores em relação a velocidade de convergência comparado com uma escolha aleatória. Propomos como vetor inicial o vetor $q_{0}=\widetilde{H}^{*} \widetilde{b}$, onde $\widetilde{b}=\left[\widetilde{h}_{j-1}, \widetilde{h}_{j}, \ldots, \widetilde{h}_{j+M}\right]^{*}$. Para explicar o motivo desta escolha, considere a decomposição em valores singulares (SVD) de $\widetilde{H}$ :

$$
\widetilde{H}=\widetilde{U} \widetilde{\Sigma} \widetilde{V}^{*}=\widetilde{\sigma}_{1} \widetilde{u}_{1} \widetilde{v}_{1}^{*}+\widetilde{\sigma}_{2} \widetilde{u}_{2} \widetilde{v}_{2}^{*}+\cdots+\widetilde{\sigma}_{N} \widetilde{u}_{N} \widetilde{v}_{N}^{*}
$$

Usando esta decomposição segue imediatamente que

$$
\widetilde{v}_{j}^{*} \widetilde{H}^{*} \widetilde{b}=\widetilde{\sigma}_{j} \widetilde{u}_{j}^{*} \widetilde{b}, \quad j=1,2, \ldots, N .
$$

Este resultado mostra que, se $\|E\| \ll \sigma_{d}$ e $\|\widetilde{b}-b\| \ll\|b\|$, então as componentes mais fortes do vetor $q_{0}$ estão associadas com os $d$ primeiros vetores singulares, os quais queremos aproximar.

\section{Experimentos Numéricos}

Para observar o desempenho do IRL em relação a escolha do vetor inicial $q_{0}$, a escolha de $p$ e do uso da multiplicação rápida, apresentamos resultados de alguns experimentos numéricos realizados com um sinal proveniente de simulações em Ressonância Magnética Nuclear [11]. O método foi programado em Matlab, e para medir o tempo computacional gasto, foi usada a função flops do Matlab. O sinal foi construído através da seguinte modelagem:

$$
\widetilde{h}_{j}=\Sigma_{k=1}^{d} a_{k} e^{i \phi_{k}} e^{\left(\alpha_{k}+i \omega_{k}\right) j \Delta t}+\epsilon_{j}, \quad j=1,2, \cdots, 512,
$$

\begin{tabular}{|c|c|c|c|c|c|c|c|c|c|c|c|}
\hline$l$ & 1 & 2 & 3 & 4 & 5 & 6 & 7 & 8 & 9 & 10 & 11 \\
\hline$a_{l}$ & 75 & 150 & 75 & 150 & 150 & 150 & 150 & 150 & 1400 & 60 & 500 \\
$\alpha_{l}$ & -50 & -50 & -50 & -50 & -50 & -50 & -50 & -25 & -286 & -25 & -200 \\
$\omega_{l} \backslash(2 \pi)$ & -86 & -70 & -54 & 152 & 168 & 292 & 308 & 360 & 440 & 490 & 530 \\
$\phi_{l}$ & 135 & 135 & 135 & 135 & 135 & 135 & 135 & 135 & 135 & 135 & 135 \\
\hline
\end{tabular}

Tabela 1: Parâmetros do sinal, onde $a_{l}$ é dada em unidades arbitrárias, $\alpha_{l}$ e $\omega_{l}$ em $\mathrm{Hz}$ e $\phi_{l}$ em graus.

onde os parâmetros $a_{l}, \alpha_{l}, \omega_{l}$ e $\phi_{l}$ representam a amplitude real, o fator de decaímento, a freqüência angular e a fase, respectivamente. Os $\epsilon_{j}$ correspondem ao ruído, o qual, 
em nossos testes, foi gerado usando o comando randn do Matlab com diferentes valores de desvio padrão $(\sigma=5,10,15)$. O sinal mostrado possui 11 exponenciais, e os parâmentros $a_{l}, \alpha_{l}, \omega_{l}$ e $\phi_{l}$ são dados na Tabela 1 .

A partir do sinal, foram geradas matrizes de Hankel de ordem $256 \times 256$, de acordo com (1.1). Na Figura 1, mostramos os 15 maiores valores singulares de $\widetilde{H}$, para dois níveis de ruído correspondentes a $\sigma=5$ e $\sigma=15$, bem como os 11 valores singulares não nulos da matriz de Hankel $H$. Observe que para $\sigma=5$, existe um gap bem notório entre $\widetilde{\sigma}_{11}$ e $\widetilde{\sigma}_{12}$. Quando o nível de ruído é aumentado para $\sigma=15$, este gap diminui, tornando o problema de calcular os 11 maiores valores e correspondentes vetores singulares mais complicado. Além disso, se o ruído introduzido for muito alto, ou seja, se $\|E\|>\sigma_{11}$, este último valor singular pode não ser recuperado.

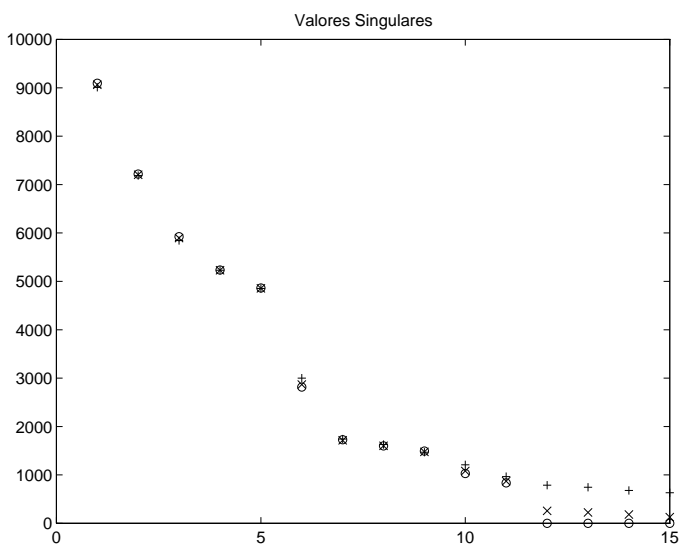

Figura 1: Em 'o' os 11 valores singulares não nulos de $H$, em 'x' e '+' os 15 maiores valores singulares de $\widetilde{H}$ para $\sigma=5$ e $\sigma=15$, respectivamente.

\begin{tabular}{|c|c|c|c|c|c|}
\hline$\sigma$ & $\mathrm{p}$ & $\begin{array}{c}\text { Recomeços } \\
q_{0} \text { aleatório }\end{array}$ & $\begin{array}{c}\text { Recomeços } \\
q_{0}=\widetilde{H}^{*} \widetilde{b}\end{array}$ & razão1 & razão2 \\
\hline \hline 5 & 5 & 3 & 0 & 3.2476 & 4.5251 \\
10 & 7 & 5 & 0 & 5.6751 & 4.2278 \\
15 & 10 & 8 & 8 & 1.0057 & 2.2862 \\
15 & 11 & 7 & 0 & 9.7371 & 3.6875 \\
\hline
\end{tabular}

Tabela 2: Comparação do número de operações de ponto flutuante para o IRL-ES, $p$ denota o número de passos Lanczos adicionais.

Para observar a performance do método em relação a escolha do vetor inicial, foram realizados testes numéricos com $q_{0}$ aleatório e com $q_{0}=\widetilde{H} * \widetilde{b}$, para vários valores de $p$. Os resultados dos experimentos são mostrados na Tabela 2, onde razão1 denota o quociente entre o número de flops gastos quando o método é inicializado 
com $q_{0}$ aleatório e o número de flops gastos quando $q_{0}=\widetilde{H}^{*} \widetilde{b}$. Analisando os resultados numéricos dessa tabela, concluímos que se o nível de ruído é alto, o valor de $p$ deve ser tomado um pouco maior do que $d$, caso contrário, o valor de $p$ é, em geral, menor do que $d$. Além disso, verificamos que a convergência é mais rápida quando o vetor inicial é escolhido como $q_{0}=\widetilde{H}^{*} \widetilde{b}$. A tabela apresenta também, na terceira coluna, a razão entre o número de flops do IRL-ES sem o uso da multiplicação rápida e com o uso da multiplicação rápida. Foram realizados os mesmos testes para outros sinais e os resultados obtidos foram similares.

\section{Conclusões}

A transformação do problema de valores singulares num problema de autovalores nos permitiu o cálculo parcial dos valores singulares, o que é muito relevante no nosso caso, pois os métodos tradicionais para o cálculo de valores singulares calculam todos os valores singulares e não levam em conta a estrutura da matriz $H$. O uso da multiplicação rápida nos permitiu explorar a estrutura da matriz $H$, tornando mais rápida a multiplicação matriz-vetor. Por outro lado, foi proposta uma escolha inicial para o processo de Lanczos, a qual aumenta a taxa de convegência em relação à escolha aleatória.

\section{Referências}

[1] F.S.V. Bazán and C. Bavastri, An Optimized Pseudo-Inverse Algorithm (OPIA) For Multi-Input Multi-Output Modal Parameter Identification, $M e$ chanical Systems and Signal Processings, 10 (1996), 365-380.

[2] F.S.V. Bazán and Ph.L. Toint, On the 2-Condition Number of Infinite Hankel Matrices of Finite Rank, "technical report 10", Department of Mathematics, FUNDP, Namur, Belgium, February 1998.

[3] F.S.V. Bazán, Conditioning of Rectangular Vandermonde Matrices with nodes in the Unit Disk, SIAM Jornal on Matrix Analysis and Applications, aparecer.

[4] D. Calvetti, L. Reichel and D.C. Sorensen, An Implicitly Restarted Method for Large Symmetric Eigenvalue Problems, Electronic Transactions on Numerical Analysis, 2 (1994), 1-21.

[5] E. Lundström, Singular Value Computations for Toeplitz Matrices, Lic. Thesis, Linköping University, ISBN 91-7871-719-1, May 1996.

[6] G.H. Golub and C.F. Van Loan, "Matrix Computations", Johns Hopkins University Press, Baltimore, third edition, 1996.

[7] P.C. Hansen, "Rank-Deficient and Discrete Ill-Posed Problems", SIAM, Philadelphia, 1997. 
[8] R.B. Lehoucq, Analysis and Implementation of an Implicitly Restarted Arnoldi Iteration. "PhD Thesis", Rice University, Houston, Texas, 1995.

[9] B.N. Parlett, "The Symmetric Eigenvalue Problem", SIAM, Philadelphia, 1997.

[10] D.C. Sorensen, Implicit Aplication of Polynomial Filters in a k-step Arnoldi Method, SIAM Jornal on Matrix Analysis and Applications, 13 (1992), 357385 .

[11] J. Totz, A.V.D. Boogaart, S. V. Huffel, D.Graveron-Demilly, I. Dologkou, R. Heidler and D. Michel, The Use of Continuous Regularization in the Automated Analysis of MRS Time-Domain Data, Jornal of Magnetic Resonance, 124 (1997), 400-409.

[12] G. Xu and T. Kailath, Fast Estimation od Principal Eigenspace using Lanczos Algorithm, SIAM Jornal on Matrix Analysis and Applications, 15 (1994), 974994. 
